\title{
Route Planning and Monitoring Design of Unmanned Aerial Vehicles Based on Global Particle Algorithm
}

\author{
Zhi Ding ${ }^{1}$ and Yuze $\mathrm{Li}^{2 *}$ \\ ${ }^{1}$ School of Computer Science and Information Engineering, Bengbu University, Bengbu 233030, China \\ ${ }^{2}$ School of Languages and Media, Anhui University of Finance and Economics, Bengbu 233030, China
}

(Received March 23, 2021; accepted June 28, 2021)

Keywords: UAVs, data transmission, cloud operating systems, route planning, multimode communications

Unmanned aerial vehicles (UAVs), possessing the characteristics of a sensor and a sensing function, are in great demand in emergency rescue, agricultural plant protection, police and law enforcement, geological exploration, environmental monitoring, film and television entertainment, and other industries. However, the UAV industry is faced with various bottlenecks, such as the low intelligence level of UAVs, the high technical threshold for completing complex tasks, the lack of autonomous cooperative ability between the formation of UAVs, and the lack of an intelligent operating system. To achieve the route planning and monitoring design of UAVs, we first designed a remote intelligent monitoring system for a UAV based on multimode communication and cloud computing, which consisted of an intelligent control module, a coordination module for the multimode communication and autonomous formation of UAVs, a UAV cloud operating system, and panoramic image dynamic fusion technology. Then, we presented an adaptive data transmission method based on priority in the multimode communication system. Finally, a route planning and monitoring design based on a global particle algorithm was designed for the remote intelligent monitoring system of the UAV. The simulation results show that the remote security system operates normally and meets urgent communication needs. At the same time, both data and video image transmission can be achieved, and the route of the UAV can be effectively planned.

\section{Introduction}

Unmanned aerial vehicles (UAVs) are aircraft that do not require a pilot to operate from the cockpit. UAV technology is a relatively new technology integrating electronic information, computer science, autonomous navigation, and other disciplines. UAVs have been widely used in military ground-to-air defense and reconnaissance, agricultural plant protection, communication transmission, forest pest control, civil three-dimensional patrol, and other fields. ${ }^{(1-3)}$ According to a research report on China's civil UAV market in 2015, agricultural applications, police/security, oil and gas pipelines, power maintenance, and other aspects will account for 68 ,

*Corresponding author: e-mail: lyz@aufe.edu.cn https://doi.org/10.18494/SAM.2021.3384 
$10,3,2$, and $17 \%$ of the future civil UAV market, respectively. Moreover, the domestic UAV market is estimated to be worth more than $\$ 7$ billion in the next five years. However, the UAV industry is faced with the following bottlenecks: the low intelligence level of UAVs, the high technical threshold for completing complex tasks, the lack of autonomous cooperative ability between the formation of UAVs, and the lack of a UAV intelligent operating system. ${ }^{(4,5)}$

Owing to the limitations of relevant technologies, research and development in the UAV remote technical support field is still insufficient. At present, there are few professional schemes for remote security design and implementation in scenarios using UAVs, which are mainly realized by obtaining the right to control a remote computer with the help of TeamViewer, PCAnywhere, and other general remote-control software, owing to relatively perfect technical applications. ${ }^{(6)}$ However, non-customizable features make remote-control software incompatible with UAVs and supporting software, and a large amount of data is transmitted. Therefore, professionalism, functionality, and real-time performance cannot meet the requirements of UAVs of high reliability and low delay. Recently, Savaglio et al. have applied cloud computing to the Internet of Things (IoT), intelligent agriculture, and the remote support or monitoring of power systems through customized development, remote monitoring, data transmission, and the monitoring of corresponding things, fully utilizing the advantages of the cloud in communication, computing, and storage. ${ }^{(7)}$ However, the existing research generally does not consider transfer problems such as real-time transfer, and the application of the cloud in the field of UAV remote technical support is still insufficient.

To resolve the above problems, we propose a remote intelligent monitoring system for UAVs based on multimode communication and cloud computing. The system consists of a UAV module, an intelligent control module (hardware), a cloud operating system (cloud software), and a display-control terminal. At the same time, a priority-based data-adaptive transmission method and route planning based on the global particle algorithm are used to realize the high-speed cloud computing and remote intelligent control of the UAV and the autonomous cooperation of intelligent UAVs during flight. In addition, we can transform and upgrade ordinary UAVs, reduce the cost of a single intelligent UAV, and reduce the flight load. Overall, this technical solution has the following potential advantages: 1) It fills the gap of UAV intelligent operating systems and overcomes the technical bottleneck. 2) It lowers the threshold of UAV operation, making UAVs easy to use and convenient. 3) The cloud operating system can be used to expand the range of applications and the market for UAVs.

\section{2. $\quad$ Related Works}

In recent years, Tange et al. have successively studied the monitoring system design of UAVs based on an STM32 single-chip microcomputer and the precoding technology in the application of the UAV communication system. ${ }^{(8)} 5 \mathrm{G}$ and UAV intelligent networking-based emergency communication technology and a wide range of UAV application scenarios, e.g., agricultural remote monitoring system design, firefighting and rescue, anti-terrorism action, astral and terrestrial collaborative intelligence, and marine communication, mean that UAV integrated technology has attracted wide attention. The key technologies of the emergency communication 
system based on 5G and UAV intelligent networking include 5G communication application technology, full-duplex two-way relay technology, directional antenna coverage technology, UAV intelligent deployment and control technology, UAV distributed intelligent networking technology, edge computing, and cloud computing technology.

To achieve civil three-dimensional safety patrol, for example, in school, college, and university campuses, national industrial parks, and residential districts, Li et al. designed a realtime video transmission system of UAV monitoring and selected a low-power STM32 singlechip microcomputer, an MPU6050 single-chip microcomputer, and a $5.8 \mathrm{GHz}$ image transmission system to realize the main control, flight control, and image transmission of the system, respectively. ${ }^{(9)}$ During navigation, a UAV can perform lifting, rolling, pitching, and yawing, and a clear real-time video can be transmitted to the monitoring module at the same time. Many tests showed that a UAV has good flight performance, can meet the requirements of the monitoring system, and can be rapidly embedded into a current monitoring system, giving it a practical value.

A UAV operates in the microwave frequency band of $3.5 \mathrm{G}$, which is used for line-of-sight communication. To realize the beyond-line-of-sight command and control of UAVs and improve the communication performance of UAVs via upstream and downstream links, multiple UAV ground stations can be networked using a ground wired network. For the downlink between a UAV and the ground station, Han et al. proposed a multiuser MIMO channel model, and multipath interference and channel noise generated by this model were mitigated by precoding technology. ${ }^{(10)}$ On the other hand, precoding techniques, such as forced zero precoding, minimum mean square error, maximum signal-to-noise ratio (MSNR), and maximum signal to leakage plus noise ratio (SLNR) criterion precoding, were analyzed and compared for different scenarios of UAV deployment.

Satisfying existing emergency communication requirements, e.g., low latency, high speed, high bandwidth, and stable connection, will enable the intelligent networking and deployment of UAVs, achieve the connectivity of 5G signals, and improve the 5G network coverage. Li et al. proposed a remote security design, implemented an emergency communication system based on $5 \mathrm{G}$ technology and UAV intelligent networking, and analyzed some of its key technologies, providing important support for improving the emergency communication reliability of rescue forces in disaster-stricken areas. ${ }^{(11)}$

Aiming at the problem of unstable data transmission of agricultural UAVs, Li et al. designed a remote monitoring system for an agricultural UAV based on distributed energy resources (DER) communication technology. This system is mainly divided into three modules, namely, the UAV, a DER grid-connected monitoring and communication system, and a ground remote monitoring system. ${ }^{(12)}$ By designing the monitoring platform of a UAV, Cintuglu et al. adopted the global particle algorithm to plan the UAV route, which enabled the real-time monitoring of the UAV flight status by a remote monitoring system. ${ }^{(13)}$ The data transmission and route planning of the UAV monitoring system were tested, and the results showed that the monitoring system can effectively plan the flight path of UAVs. 


\section{Remote Intelligent Monitoring System}

The proposed UAV remote intelligent monitoring system architecture based on cloud computing is composed of UAVs, an intelligent control module, a cloud operating system, and user terminals as shown in Fig. 1. The intelligent control module is installed on the UAV and uses multimode communication technology to ensure a stable connection between the UAV and the cloud server. The cloud operating system, installed on the cloud server, undertakes the realtime processing of UAV communication, flight control, formation management, and monitoring information. The control terminal displays the dynamic panoramic map processed by the cloud operating system and receives user instructions to remotely control the UAV to perform tasks.

\subsection{Intelligent control module}

An ordinary UAV should be equipped with an intelligent control module, which achieves UAV image transmission and flight control. On the one hand, aerial images from the UAV are transmitted to cloud servers. On the other hand, the UAV is controlled through its flight control interface. The multimode communication technology is used to ensure a stable connection between the UAV and the cloud server.

\subsection{Coordination of multimode communication and autonomous formation}

The multimode communication system ensures multimode communication and the connection with the cloud operating system, e.g., $\mathrm{WiFi}+4 \mathrm{G}$, and provides collaborative signals for the autonomous formation of UAVs via the Zigbee protocol.

\subsection{UAV cloud operating system}

Cloud computing is Internet-based computing, with the advantages over traditional computing services of flexible expansion, flexible access to resources, convenient monitoring,

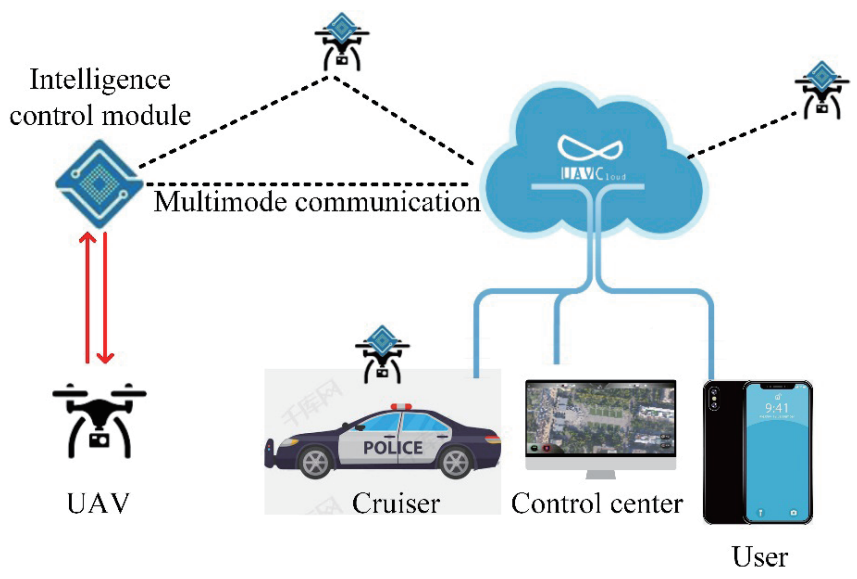

Fig. 1. (Color online) Remote intelligent monitoring system of UAV based on cloud computing. 
stability, and reliability. It is widely used in communication technology and can provide remote technical support with high-speed network transmission, high efficiency, and low running cost. This paper presents the stable and reliable Ali cloud computing platform as the server development platform. The Sky Eye Guard UAV cloud operating system is capable of high-speed cloud computing and real-time processing of several complex tasks, including panoramic image dynamic fusion, ultrahigh-resolution visual dynamic target detection, and the formation of remote coordination UAVs, as well as rapid forensics and the intervention of remote-control UAVs. We analyze the environment surrounding the UAV, intelligently control the UAV according to the analysis results, and provide a UAV three-dimensional system for patrolling campuses, businesses, and communities. The cloud operating system is used to analyze highaltitude aerial photos in real time, identify and track suspicious people, and highly integrate the autonomous formation of UAVs with existing patrol cars.

\subsection{Panoramic image dynamic fusion technology}

Panoramic image dynamic fusion technology can be installed on an ordinary patrol car or connected to a central control center and has the following functions: display integrated highdefinition panoramic information, activate a dynamic abnormality alarm, rapidly set the formation of UAVs, remotely control the UAV for on-site intervention, obtain evidence of suspicious personnel, issue warnings, and so forth.

\section{Priority-based Adaptive Data Transmission Method}

\subsection{Data priority analysis}

The transmission data in the multimode communication system can be divided into the following three categories: control command data $I_{c}$, flight status data $I_{f}$, and system operation data $I_{s}{ }^{(14,15)}$ If the transmission is in a mixed-generation sequence, the waiting time for important data before the transmission will be extremely long, which will affect the real-time performance of the system. To ensure the high transmission performance of important data, it is necessary to assign the data priority according to real-time requirements and use different queues for transmission. $I_{c}$ comprises the artificially generated flight control, load control, and other instructions. Owing to the control characteristics and requirements of UAVs, real-time requirements have the highest priority. $I_{f}$ comprises the flight status, load status, and other data of the UAV, and its real-time requirement is second only to $I_{c}$. $I_{S}$ comprises the operation data of the remote technical support system, which has no direct correlation with the flight mission and has the lowest real-time requirement. Thus, the priority of data transmission can be defined as $I_{c}>I_{f}>I_{s}$

\subsection{Design of transmission protocol}

According to the characteristics of the adaptive data, a transmission control protocol (TCP) for data instruction transmission and a hypertext transport protocol (HTTP) for operation 
information transmission are designed. The former protocol is used to transfer $I_{c}$ and $I_{f}$. As shown in Table 1, the designed transmission protocol includes six parts: a frame header, frame length, type number, data content, check bit, and frame tail. Among them, the frame head, frame length, and frame tail guarantee the integrity of a single packet in TCP streaming transmission, and the check bit guarantees correctness. An identity card (ID) is used to distinguish the type of protocol, and the data content is used to carry data or instructions. The transmission protocol uses the request-response model of the HTTP and is simple, flexible, and connectionless. In the protocol, the uniform resource location (URL) is used to represent the types of login, connections, and other operations, and the request body is used to carry $I_{s}$.

\subsection{Design of adaptive transmission algorithm}

The priority transmission method ensures the optimal transmission of important data, ${ }^{(16)}$ but when the system maintains multiple remote technical support groups, the single available bandwidth $b$ will be reduced, resulting in increased transmission delay and even congestion, which has a particularly significant impact on low-priority data. To complete the transmission of all data within the time slot $T$, the following formula must be satisfied:

$$
I_{c}+I_{f}+I_{s} \leq b \times T .
$$

The analysis of data transmission further shows that $I_{c}$ and $I_{S}$ are generated by the human operation. Since the human operation has mutability and continuity when and after data transmission occurs, respectively, the data volume of the next time slot can be estimated from the data transmission of the historical time slot after the latest mutation. $I_{f}$ represents the analog-to-digital conversion (ADC) of flight control acquisition, and the adaptive data is generated at a certain frequency $f_{u}$. According to experience, $f_{u}$ can be adjusted within $5-50 \mathrm{~Hz}$. Therefore, the frequency $f_{u}$ can be adjusted by calculating the available bandwidth and estimating the amount of $I_{c}$ and $I_{S}$ to optimize the bandwidth utilization and ensure the reliable and realtime transmission of secondary data. The cloud server's fixed bandwidth is $B$, the available bandwidth coefficient is $\beta$, and the number of remote support groups is $m$. The system adopts the average bandwidth allocation strategy, then the available bandwidth $b$ of a single remote support group is calculated as

$$
b=\frac{\beta \cdot B}{m} .
$$

The amount of $I_{f}$ in the next time slot can be calculated as

$$
I_{f}=f_{u} \cdot d_{u} \cdot T
$$

Table 1

Data instruction protocol frame structure in multimode communication.

\begin{tabular}{cccccc}
\hline Frame head & Frame length & ID & Data content & Check bit & Frame tail \\
\hline
\end{tabular}


where $d_{u}$ is the volume of a single packet of $I_{f}$, which is determined according to the transmission protocol. When estimating the amounts of $I_{c}$ and $I_{s}$ in the next time slot, the calculation weight of the data sent in the previous time slot is set as $w_{i}>0(i=1,2, \ldots, n)$. The greater the value of $i$ is, the smaller the value of $w_{i}$ is, and $\sum_{i=1}^{n} w_{i}=1$. To improve the efficiency of the algorithm, we set $w_{1}>w_{2}=\ldots=w_{n} . I_{i}$ is the actual amount of data sent in the past time slot $i$, and $I^{1}$ is estimated as

$$
I^{1}=\sum_{i=1}^{n} w_{i} \cdot I_{i}
$$

where $I_{i}=q_{i} \cdot d, q_{i}$ being the number of packets recorded on the server side and $d$ the volume of a single packet of both $I_{c}$ and $I_{s}$. The key factors to solving the above equation lie in the number of effective slots, $n$, after the last mutation and the calculated weight $w_{i}$. Taking $I_{s}$ as an example, the relative change in the volume of data between time slots is defined as

$$
\operatorname{per}=\frac{q_{0}-q_{1}}{\operatorname{Max}\left(q_{0}, q_{1}\right)}
$$

When per is greater than the mutation threshold $\operatorname{per}_{M}$, i.e., a data volume mutation occurs, and the number of time slots recorded is $n, w_{1}$ is set as $W \in(1 / n, 1)$, then the weights of the other time slots after the last mutation are $w_{i}=(1-W) /(n-1)$. According to the number of packets sent by the client record, $p_{i}$, and the number of packets received by the server record, $q_{i}, S_{i}$ is used to indicate whether the transmission of the $i$ th time slot in the past has been completed. If the transmission has been completed, $S_{i}$ is 1 ; otherwise, it is 0 , i.e.,

$$
S_{i}= \begin{cases}0, & \text { if } q_{i}>p_{i}, \\ 1, & \text { if } q_{i}<p_{i} .\end{cases}
$$

Suppose that the impact factor of a completed transmission is estimated to be $k_{s}$, the impact factor of an incomplete transmission is $1-k_{s}$. Then, the weight calculation formula is

$$
\begin{aligned}
& w_{1}=k_{s} \cdot S_{1} \cdot W+\left(1-k_{s}\right) \cdot\left(1-S_{1}\right) \cdot W, \\
& w_{i}=\frac{k_{s} \cdot S_{i} \cdot\left(1-S_{1} W\right)}{\sum_{i=2}^{n} S_{i}}+\frac{\left(1-k_{s}\right) \cdot\left(1-S_{i}\right) \cdot\left(1-S_{1} W\right)}{\sum_{i=2}^{n}\left(1-S_{i}\right)} .
\end{aligned}
$$

Similarly, since the priority of $I_{c}$ is the highest, $S_{i}$ is equal to $1, k_{s}$ is equal to 1 , and its calculated weight is $w_{1}=W, w_{i}=(1-W) /(n-1)$. The priority-based UAV data-adaptive transmission algorithm is shown in Algorithm 1. 
Algorithm 1

Priority-based UAV data-adaptive transmission algorithm.

Step 1: Determine the server fixed bandwidth $B$ and single-packet data volumes $d_{c}, d_{u}, d_{s}$, and initialize parameters such as $\beta, W$, per $_{M}, k_{s}, f_{u}$, and $T$.

Step 2: Preprocess the generated packets according to the priority order and constraint $f_{u}$, place them in the corresponding transmission queue, and mark the serial number.

Step 3: Transmit data, record the packet numbers $p_{i}$ and $q_{i}$ of the three queues in $T$, then calculate the relative changes in data volume $\operatorname{per}_{c, i}$, per $_{s, i}, S_{c, i}$, and $S_{s, i}$ using Eqs. (5) and (6), and update the corresponding $n_{c}$ and $n_{s}$.

Step 4: Calculate the past slot weights $w_{c, i}$ and $w_{s, i}$ of $I_{c}$ and $I_{s}$, respectively, using $S_{c, i}, S_{s, i}, n_{c}, n_{s}$, and Eq. (7).

Step 5: Calculate the data of the next time using $w_{c, i}, w_{s, i}, q_{c, i}, q_{s, i}$ and Eq. (4), and estimate $I_{c}^{1}$ and $I_{s}^{1}$.

Step 6: Obtain the current number of online remote support groups, $m$, and calculate the single available bandwidth $B$ using Eq. (2).

Step 7: Calculate the maximum allowable amount of $I_{f}^{1}$ for the next time slot using $b, I_{c}^{1}, I_{s}^{1}$, and Eq. (1).

Step 8: Calculate $f_{u}$ of the next time slot using $I_{u}, d_{u}$, and Eq. (3). If $f_{u}<5 \mathrm{~Hz}$, then go to Step 9, otherwise return to Step 2.

Step 9: Set $f_{u}=5 \mathrm{~Hz}$. If $f_{u}$ cannot meet the task requirements, increase the time slot to $T=(1+\lambda) T$, where $\lambda \in(0,1)$ is the time slot adjustment factor. Return to Step 2.

\section{Route Planning and Monitoring Design Based on Global Particle Algorithm}

The remote monitoring system of the UAV is mainly used to monitor and control its flight status. One of the main aspects of monitoring a UAV is to plan its route for different tasks, mainly considering the following constraints: (1) The UAV can only fly in civil areas and must not enter no-fly zones. (2) Owing to the limitation of the mobility of the UAV, it is necessary to keep an appropriate distance between the UAV and obstacles and the ground to avoid accidents. (3) The pitching and deviation angles during UAV flight should be within the range of UAV performance constraints. A schematic diagram of the pitching and deviation angles is shown in Fig. 2.

It can be seen from Fig. 2 that $\beta$ represents the pitching angle, and the deviation angle $\gamma$ range is expressed by the formula $\left(a_{i}^{T} a_{i+l}\right) /\left(\left|a_{i}\right|+\left|a_{i+l}\right|\right) \geq \cos \gamma_{\max }$, where $a_{i}$ is the projection of the track of stage $i$ of the UAV on the horizontal plane. The pitching angle range can be expressed as $\left|z_{i}-z_{i-l}\right| /\left|a_{i+l}\right| \leq \tan \beta_{\max }$, where $z_{i}$ is the coordinate value of the track of section $i$ on the $z$-axis. Since the UAV is battery-powered, it is necessary to ensure that it travels within the maximum range, and the formula used to calculate the remaining battery capacity is

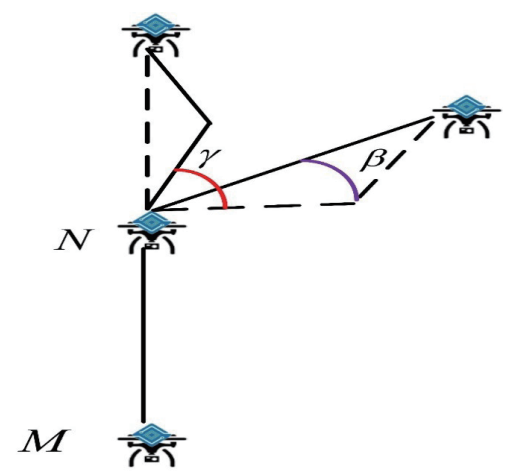

Fig. 2. (Color online) Architecture of UAV pitching and deviation angles. 


$$
v_{u n}=\frac{2}{\left|2-\varphi-\sqrt{\varphi^{2}-4 \varphi}\right|}\left[v_{i j}+a_{1} b_{1}\left(p_{i j}-x_{i j}\right)+a_{2} b_{2}\left(p_{u n}-x_{u n}\right)\right],
$$

where $\varphi=a_{1}+b_{1}, a_{1}=a_{2}=2.05$, and $p_{i j}$ and $p_{u j}$ are the optimal positions of particles in the $j$-dimensional space. To accelerate the convergence of particles, a linearly decreasing weight is set for the particle velocity weight, i.e., $\sum_{i=1}^{n} \sqrt{\left(x_{i+1}-x_{i}\right)^{2}+\left(y_{i+1}-y_{i}\right)^{2}+\left(z_{i+1}-z_{i}\right)^{2}} \leq S_{m a}$, where the track coordinate value of section $i$ is $x_{i}$ and the maximum range is $S_{\max }$. The global particle algorithm is used to plan the flight path of a UAV, which is characterized by simple calculation and easy implementation. The implementation steps are shown in Fig. 3.

The position of particle $i$ is $X_{i}=\left(x_{i 1}, x_{i 2}, \ldots, x_{i s}\right)$, the optimal position of particle $i$ is $P_{i}=\left(p_{i 1}, p_{i 2}, \ldots, p_{i s}\right)$, and the global optimal position of particle $i$ is denoted as $P_{u}=\left(p_{u 1}, p_{u 2}, \ldots, p_{u s}\right)$. Here, $u$ is used to index the optimal position of each particle. The general global particle algorithm adjusts its position through the following formula to confirm its own position and the global optimal position, i.e.,

$$
v_{i j}^{k+1}=\omega \cdot v_{i j}^{k}+a_{1} b_{1}\left(p_{i j}-x_{i j}^{k}\right)+a_{2} b_{2}\left(p_{i j}-x_{i j}^{k}\right) .
$$

where $v_{i j}^{k}$ defines the velocity of particle $i$ in $j$-dimensional motion after $k$ iterations, $\omega$ denotes the particle velocity weight, both $a_{1}$ and $a_{2}$ are the acceleration factors of particles, as well as $b_{1}$ and $b_{2}$ are random values in the range of $[0,1]$. In the application of this algorithm, there are some problems such as particle collision and trapping at local optima, which should be solved. The iteration speed of each particle is adjusted to make its range less than the maximum traveling speed so that the optimal solution is searched for within a fixed range. The iteration speed is calculated as

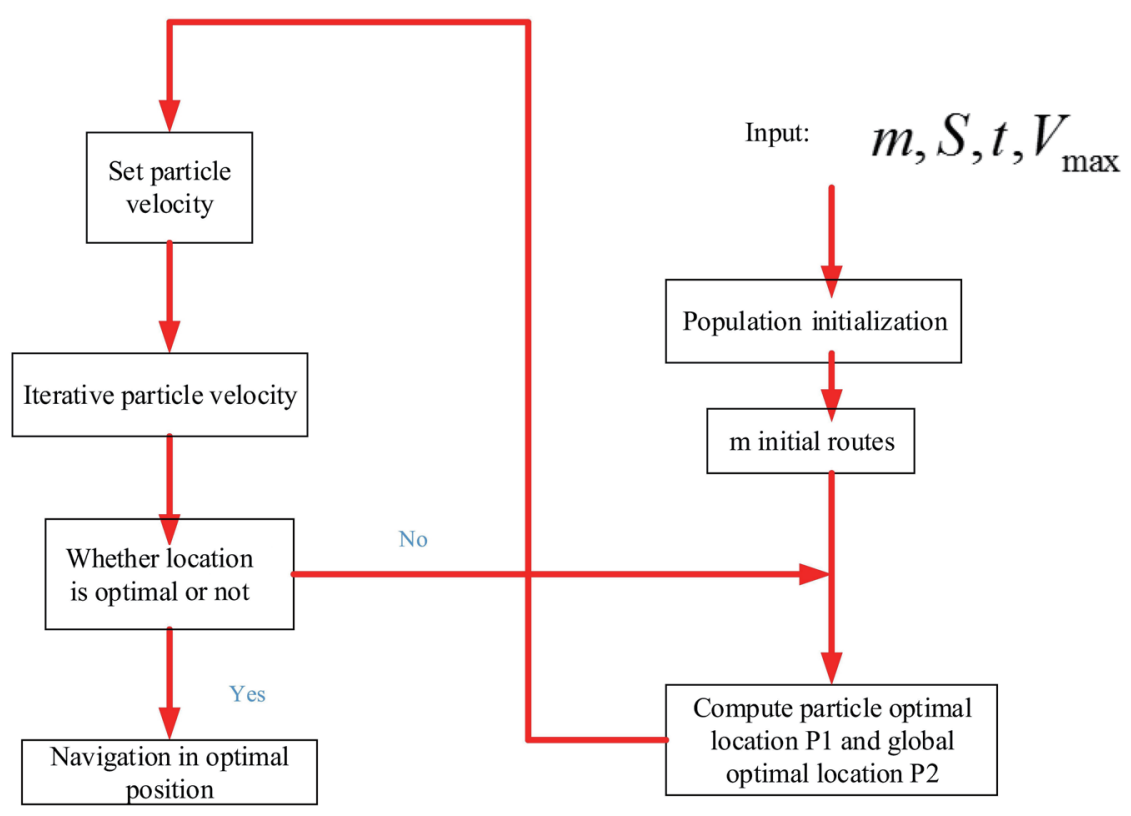

Fig. 3. (Color online) Flow chart of global particle property algorithm. 


$$
\omega=\omega_{\min }+\frac{\omega_{\max }-\omega_{\min }}{l}(l-k),
$$

where $\omega_{\min }$ and $\omega_{\max }$ are the minimum and maximum inertia coefficients of the particle, respectively, $l$ is the total number of particle velocity iterations, and $k$ is the current number of particle iterations.

\section{Results and Discussion}

The UAV ground station software is launched on two computers: one as the supporter client and the other as the recipient client. After registering an account, we log into the server, select the recipient client to connect, and start the remote technical support. The ground station software on the supporter side can observe the flight attitude, position, track point, and other data consistent with those on the recipient side. The remote UAV can receive and execute debugging parameters and takeoff commands sent by the supporter client. The simulation results show that the system runs stably, achieves the target function, and meets professional and functional requirements.

In this experiment, the sending time SendTime and receiving time RecvTime of 5000 protocol packets are recorded with the DateTime function on the supporter and recipient clients. The results of calculating the transmission time of each packet are as follows: the maximum time is $36 \mathrm{~ms}$, the minimum time is $9 \mathrm{~ms}$, and the average time is $13 \mathrm{~ms}$. As shown in Fig. 4, the transmission time for 100 consecutive packets can be seen by drawing a line graph, which shows that the time is relatively stable. Since the shortest response time of the continuous operation software is several hundred milliseconds, which is longer than the transmission time, the transmission negligibly affects the operation delay and meets the real-time requirements.

To simulate multigroup remote technology to support concurrency, the available bandwidth was limited to $15000 \mathrm{bit} / \mathrm{s}$ in the experiment, and the algorithm parameters were initialized, in which the time slot $T$ is $1 \mathrm{~s}$ and the initial frequency $f_{u}$ of $I_{u}$ is $25 \mathrm{~Hz}$. The same operation is repeated with and without the priority-based UAV data-adaptive transmission method. As shown in Fig. 5, when the bandwidth is limited, this method automatically adjusts $f_{u}$ as $I_{c}$ and $I_{s}$ change.

A comparison of the transmission success rates is shown in Table 2. The transmission success rate is calculated as the ratio of the number of packets received in a slot to the number of packets

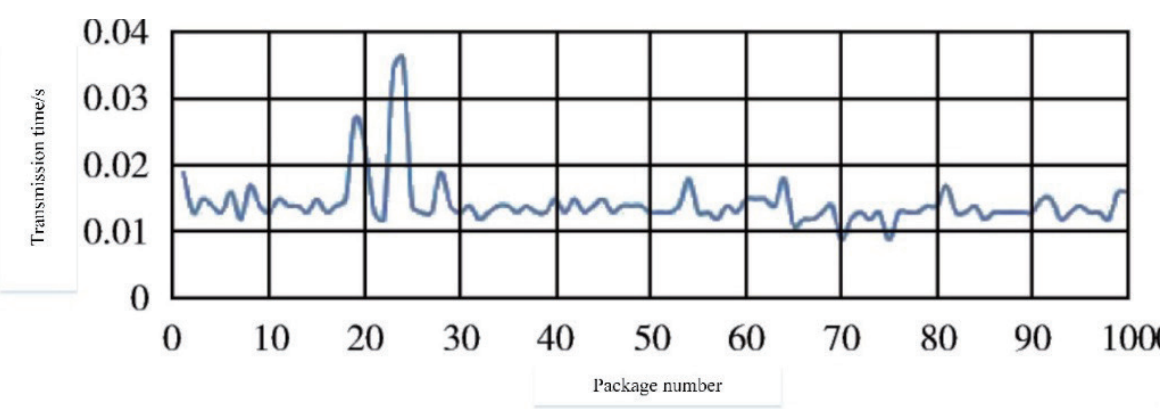

Fig. 4. (Color online) Transmission time variation. 


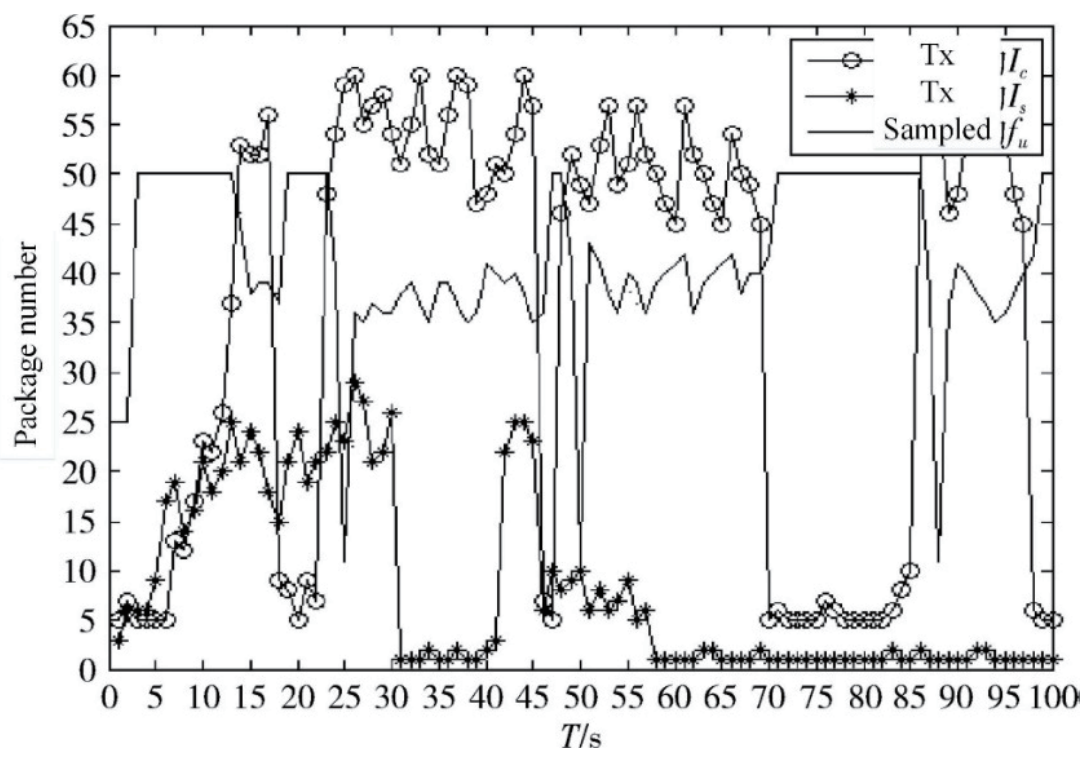

Fig. 5. Effect of data frequency adjustment.

Table 2

Comparison of transmission success rates.

\begin{tabular}{lcc}
\hline Solution parameters & Unenabled method $^{(16)}$ & Enabled method (proposed) $^{(100}$ \\
\hline$I_{c}$ & 73.56 & 100 \\
$I_{u}$ & 28.91 & 100 \\
$I_{S}$ & 80.22 & 93.25 \\
All & & 99.00 \\
\hline
\end{tabular}

sent. The average transmission time of each queue and all data is calculated. Compared with that of the unenabled method, the transmission time of this method is small, particularly for lowpriority data. The results show that the data transmission performance can be guaranteed and the real-time performance can be satisfied by adjusting the data frequency when the available bandwidth is reduced owing to multiple groups of concurrencies.

The test site in the experiment was a school playground. We ensured that no other UAV was flying in the site during the test, and we collected and saved the test data. The two main aims of the test were to evaluate the data transmission and carry out the route planning of a UAV using the UAV remote monitoring system. The UAV was manually operated according to the preset trajectory and it was observed in real time whether the transmitted video images could be observed on the display. All flight information of the UAV was configured to carry out route planning in an area of $100 \times 100 \mathrm{~m}^{2}$. Three no-flight zones were set inside the test site, i.e., a $5 \times$ $5 \times 5 \mathrm{~m}^{3}$ cubic zone in the northeast, a $5 \times 5 \times 5 \mathrm{~m}^{3}$ cubic zone in the southwest, and a spherical zone of $5 \mathrm{~m}$ diameter in the center. The cubic obstacles were set in the test site to make the system automatically plan the UAV's track point. The track point results obtained after route planning are shown in Fig. 6. The positions of the track points avoid the no-fly zones and obstacles, indicating successful route planning. 


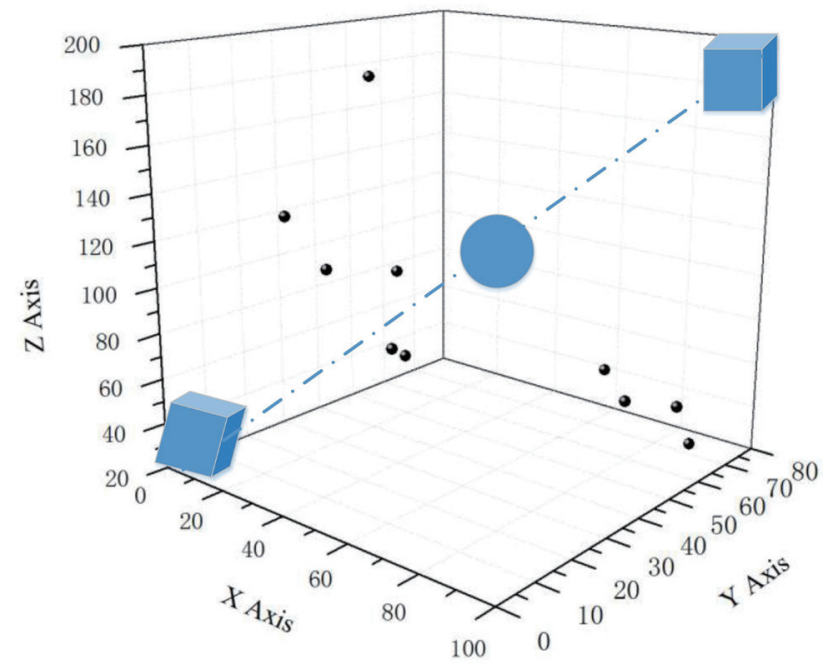

Fig. 6. (Color online) Track point results after route planning.

\section{Conclusions}

The remote monitoring system of a UAV was designed using multimode communication technology in this study. The system is mainly divided into four modules, i.e., an intelligent control module, a multimode communication and autonomous formation collaboration, a UAV cloud operating system, and panoramic image dynamic fusion technology. The monitoring platform of the remote monitoring system of a UAV was designed, and the flight path of the UAV was planned by using the global particle algorithm to ensure the real-time monitoring of the flight status of the UAV by the remote monitoring system. The data transmission and flight path planning of the UAV monitoring system were tested. The results show that the monitoring system can complete the transmission of data and video images and carry out effective track planning for UAVs. In addition, a cloud-computing-based UAV remote technical support system was designed and implemented. We customized the development of the cloud server and client, using the cloud and the network for long-distance communication, and achieved flight monitoring, parameter adjustment, and other remote support functions. According to the characteristics of data transmission, an adaptive data transmission method based on priority was also proposed for UAVs. In contrast to the conventional transmission method, the transmission performance of important data is guaranteed, and the ability to perform real-time transmission and the transmission reliability are improved in the case of reduced available bandwidth. Through an actual flight experiment, it was verified that the system runs normally and meets the functional and performance requirements.

\section{Acknowledgments}

This work was supported by the Mixed Online and Offline First-class Course Project of the Ministry of Education "Foundation and Application of Multimedia Creation" (2020140423) and 
the New Engineering Construction Project of Bengbu University on the Training Mode of New Engineering Talents Based on Huawei ICT College (2020XGKJY9).

\section{References}

1 J. Kim, S. Kim, C. Ju, and H. I. Son: IEEE Access 7 (2019) 105100. https://doi.org/10.1109/ ACCESS.2019.2932119

2 M. N. Boukoberine, Z. Zhou, and M. Benbouzid: Appl. Energy 255 (2019) 113823. https://doi.org/10.1016/j. apenergy.2019.113823

3 S. Park and Y. Choi: Minerals 10 (2020) 663. https://doi.org/10.3390/min10080663

4 S. Aggarwal and N. Kumar: Comput. Commun. 149 (2020) 270. https://doi.org/10.1016/j.comcom.2019.10.014

5 Y. Liu, X. Nie, J. Fan, and X. Liu: Comput.-Aided Civ. Inf. 35 (2020) 511. https://doi.org/10.1111/mice.12501

6 A. Nauman, Y. A. Qadri, and M. Amjad: IEEE Access 8 (2020) 8202. https://doi.org/10.1109/ ACCESS.2020.2964280

7 C. Savaglio, M. Ganzha, and M. Paprzycki: Future Gener. Comput. Syst. 102 (2020) 1038. https://doi. org/10.1016/j.future.2019.09.016

8 K. Tange, M. De Donno, X. Fafoutis, and N. Dragoni: IEEE Commun. Surv. Tutorials 22 (2020) 2489. https:// doi.org/ 10.1109/COMST.2020.3011208

9 H. Li, M. K. Ochani, H. Zhang, and L. Zhang: J. Eng. 13 (2017) 1629. https://doi.org/10.1049/joe.2017.0608

10 S. Han, Y. Zhang, W. Meng, and H. Chen: IEEE Trans. Veh. Technol. 67 (2018) 8249. https://doi.org/10.1109/ TVT.2018.2840824

11 B. Li, Z. Fei, and Y. Zhang: IEEE Internet Things J. 6 (2019) 2241. https://doi.org/10.1109/JIOT.2018.2887086

12 B. Li, Z. Fei, Y. Zhang, and M. Guizani: IEEE Wireless Commun. 26 (2019) 114. https://doi.org/10.1109/ MWC.2019.1800458

13 M. H. Cintuglu, H. Martin, and O. A. Mohammed: IEEE Trans. Smart Grid 6 (2015) 1064. https://doi. org/10.1109/TSG.2014.2387215

14 R. Chen, W.-X. Long, and X. Wang: IEEE Trans. Wireless Commun. 19 (2020) 6932. https://doi.org/10.1109/ TWC.2020.3007026

15 X. Ding, F. Ding, X. Chen, and X. Wang: IET Commun. 14 (2020) 1221. https://doi.org/10.1049/ietcom.2019.0117

16 J. Zhu, X. Jiang, Y. Yu, G. Jin, H. Chen, X. Li, and L. Qu: China Commun. 17 (2020) 37. https://doi. org/10.23919/JCC.2020.06.004

\section{About the Authors}

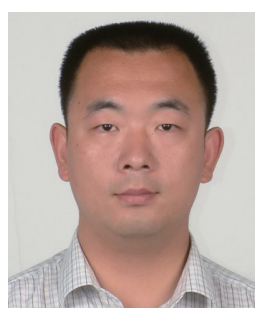

Zhi Ding received his B.S. degree in 2004. He is currently a senior engineer at the School of Computer Science and Information Engineering, Bengbu University. His research interests include network security and network applications.

(dingzhibb@163.com)

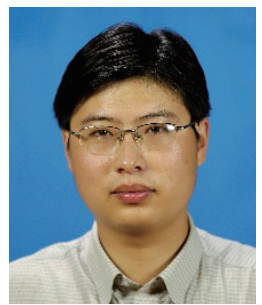

Yuze Li, born in Bengbu, Anhui Province, received his B.S. degree from Anhui Normal University, China, and his M.S. degree from Nanjing Normal University, China, in 2001 and 2008, respectively. Currently, he is a Ph.D. student at the Assumption University, Thailand. He is a senior experimentalist at the School of Languages \& Media, Anhui University of Finance and Economics. His research interests include the theory and practice of educational technology. (lyz@aufe.edu.cn) 